\title{
Dans le signe du son. Bruit, voix, corps et improvisation
}

\section{Davide Sparti}

Traducteur : Giuliana Ravviso

\section{OpenEdition \\ Journals}

Édition électronique

URL : http://journals.openedition.org/imagesrevues/417

DOI : 10.4000/imagesrevues.417

ISSN : 1778-3801

\section{Éditeur :}

Centre d'Histoire et Théorie des Arts, Groupe d'Anthropologie Historique de l'Occident Médiéval, Laboratoire d'Anthropologie Sociale, UMR 8210 Anthropologie et Histoire des Mondes Antiques

\section{Référence électronique}

Davide Sparti, « Dans le signe du son. Bruit, voix, corps et improvisation », Images Re-vues [En ligne], 7| 2009, document 9, mis en ligne le 21 avril 2011, consulté le 30 janvier 2021. URL : http://

journals.openedition.org/imagesrevues/417 ; DOI : https://doi.org/10.4000/imagesrevues.417

Ce document a été généré automatiquement le 30 janvier 2021.

\section{(c) (1) 8}

Images Re-vues est mise à disposition selon les termes de la Licence Creative Commons Attribution Pas d'Utilisation Commerciale 4.0 International. 


\section{Dans le signe du son. Bruit, voix, corps et improvisation}

\section{Davide Sparti}

Traduction : Giuliana Ravviso

"Yes, I really like things that are imperfect, instead of perfect" Steve Lacy

" utterly unheard sounds, the unheard of sounds

Steve Lacy 
1 Si l'on considère l'histoire de la musique, il est étonnant de constater que la musique baroque y opère, par son absence d'œuvres, une rupture radicale ; en effet, ce qui caractérise une cuvre - à savoir qu'elle est instrumentale, transcrite, indépendante, selon la définition de Dahlhaus $^{1}$ - ne peut lui être attribuée. On a affaire à des pièces, à des stücke, mais

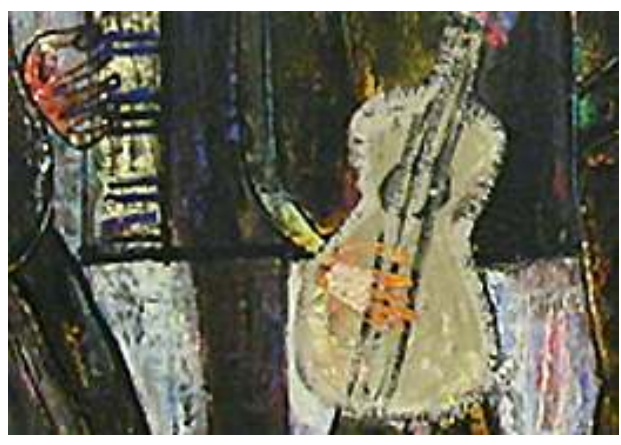
non à des œuvres autonomes auxquelles pourrait être attribuée la valeur d'entités achevées. Certes, il existe des partitions, mais l'écriture musicale, qui n'a pas encore trouvé sa spécificité, est utilisée de façon pragmatique, c'est-à-dire qu'elle se caractérise par l'ornementation, l'improvisation, et une relative fluidité entre ce qui est propre à la composition et ce qui tient à l'exécution. La musique est davantage conçue comme un support et un complément plutôt que comme une forme artistique : elle accompagne la poésie, le théâtre, la liturgie, le mariage.

2 L'idée qu'une œuvre doit être composée à des fins purement instrumentales, qu'elle est une création figée, indépendante de son exécution, qui sera jouée régulièrement et sur une longue période et dans des lieux différents, est absente ou presque des attentes d'un public qui chaque fois s'attend à un événement musical nouveau.

3 Rappelons-nous d'ailleurs que tous les textes des cantates de J.S. Bach ont été publiés de son vivant, mais la musique pour deux entre elles seulement. Ce qui démontre que l'idée d'une œuvre instrumentale autonome ne s'était pas encore affirmée. Composer de la musique pour la postérité (de la musique qui serait reproduite) n'était pas le souci majeur des musiciens. Au cours de la période romantique, la situation change radicalement, bien que de façon progressive. C'est pendant cette période en effet que s'achève le processus de séparation structurelle entre d'une part la composition (fixée par l'écriture, conçue pour la durée, titrée, liée à un créateur donné, sujette à des droits d'auteur mais aussi au plagiat) et d'autre part l'exécution. L'œuvre peut être exécutée indéfiniment et indépendamment de son compositeur. La musique devient un produit de l'écriture - la partition est détaillée et l'exécution se fait fidèle et intégrale (le terme Werktreu, fidélité à la partition, met l'accent sur l'inviolabilité du texte écrit). L'œuvre désormais coïncide avec un texte pré-écrit et composé pour la répétition, un texte qui permet que le public puisse réécouter et non pas simplement écouter des œuvres musicales dans le silence des salles de concert. Comment une telle révolution s'explique-t-elle? Quelle est sa signification culturelle?

\section{Vidéocentrisme et paradigme rétinien}

4 Pour expliquer un tel tournant, nous pouvons nous référer à Hans Jonas ${ }^{2}$ : les sons et l'ouïe sont caractérisés par l'évanescence, ils ne se manifestent qu'en se soustrayant, en passant. En ce qui concerne la musique, l'objet acoustique est un événement temporel doté d'une nature processive : il dure tant qu'il est perçu (ou reproduit mentalement, mais là aussi l'objet acoustique n'a qu'une consistance temporelle). Le son n'est pas un objet, ni ne le révèle, mais il est quelque chose qui arrive, un événement. En somme, l'ouie ne peut se détacher de la dynamique d'un flux : je dis que j'entends un chien, 
mais ce que j'entends est un chien qui aboie. Les choses ne sont pas audibles de la même manière qu'elles sont visibles : il ne leur appartient pas d'émettre des sons comme il est de leur nature immanente de réfléchir la lumière ; quelque chose doit les faire résonner et les rendre perceptibles. La vue, au contraire, saisit les objets marqués par la présence et la permanence, ouvrant un présent continu, étendu, qui n'est pas seulement l'expérience ponctuelle du maintenant mais une dimension qui nous permet de retenir les choses et de les rendre durables (à condition qu'elles ne se modifient pas).

Choisissons un exemple relatif à cette distinction entre l'espace et le temps, la vue et l'ouie, l'image et le son : si je regarde un tableau dans un musée, puis je sors et je reviens une heure plus tard le revoir, il ne me vient pas à l'idée de me demander si le tableau pourrait, entre temps, s'être modifié, bien que des modifications au niveau microphysique ne sont pas à exclure. En revanche, la musique et les autres arts performatifs sont indissociables de leur réception dans le temps, puisque précisément il s'agit d'événements devant se dérouler dans un intervalle de temps. Il est donc nécessaire, lorsqu'on assiste à une performance musicale, d'en suivre le déroulement du début à la fin, sans la fractionner, à moins de mettre en péril la performance ellemême. La durée de la performance n'est pas une question de persistance mais de processus, d'un processus irréversible et, en principe, qui ne se reproduira pas. En d'autres mots, une performance n'est jamais complètement présente dans chaque fraction de temps. Alors que les éléments d'un tableau existent de façon simultanée et que ce tableau est présent pour nous dans sa totalité, dans chaque fragment du temps de son existence. Mais le son est un flux et pour le conserver, il ne peut être "arrêté " comme un photogramme de film. Une fois qu'il cesse, il ne reste que du silence (nous sommes hors du monde du son). La vue, en revanche, saisit aussi bien le mouvement que l'immobilité. Je peux arrêter l'image et « l'avoir » en même temps (en gardant le cadrage sur l'écran). En outre, la vue préfère la fixité : en effet, pour pouvoir analyser une chose attentivement, il est préférable qu'elle soit immobile ${ }^{3}$. Pour reprendre notre affirmation précédente, dans son assujettissement aux pratiques fondées sur l'écriture, la musique se délivre de la temporalité (et de la "marginalité », qui est surtout celle du musicien par rapport à ses collègues poètes, peintres ou architectes dont le rôle social était célébré depuis l'Antiquité, il n'est que de se rappeler le fameux portrait peint de Joseph Haydn portant la livrée de la famille Esterházy). En tant que composition transcrite, la musique devient un produit durable à l'égal des autres beaux-arts. La partition a, en effet, quelque chose de dur, de matériel, de marmoréen, de monumental, en somme elle a un poids particulier que le son seul ne possède pas.

Dans l'usage que je fais de catégories d'époques si tranchées, je ne voudrais pas donner l'impression que ma lecture soit panhistorique : la généalogie est une méthode qui précisément permet d'avancer et de reculer vis-à-vis d'une problématisation valable aujourd'hui. Il faut donc répéter le point fondamental : la musique, art du temps, est de manière constitutive indissociable de l'acte performatif et disparait sans laisser des traces. D'où cette nature éphémère de la musique, et même caduque comme l'a souligné Léonard de Vinci : «Adonque la musica, che si va consumando mentre ch'ella nasce, è men degna che la pittura $»^{4}$. La musique en tant que moyenartistique n'a pas la capacité de se constituer en forme d'œuvre stable et durable comme les chefs-d'œuvre des autres beaux-arts. Ainsi, Léonard de Vinci exaltant l'autocombustion de l'élément sonore et hiérarchisant implicitement les arts, affirme : "Ma la pittura eccelle e signoreggia la musica perché essa non muore immediatamente dopo la creazione, come 
fa la sventurata musica, anzi resta in essere, e ti si dimostra in vita quel che in fatto è una sola superficie $»^{5}$. Peut-être n'est-ce donc pas un hasard si, quelques années plus tard, en 1556, Hermann Finck va considérer la musique - transcrite sur papier - comme un monumentum dans le sillage des Vite de Vasari. Pour imiter les autres arts, en particulier la peinture et la sculpture, et pour être conservée comme " objet ", la musique a alors dû valoriser non pas l'aspect " exécution ", mais quelque chose qui serait tangible, qui aurait la solidité d'un produit visible et durable - dans une culture vidéocentriste ou " rétinienne ", l'être coïncide d'abord avec l'être vu'.

7 Cela est d'autant plus vrai pour la musique improvisée dont la nature éphémère se radicalise. Tandis que le compositeur crée de la musique de façon discontinue « en dehors " du flux temporel, celui qui improvise exécute une composition qui prend forme pendant son exécution même, sans l'aide de quelque dispositif lui indiquant quelles notes jouer et dans quel ordre. Il y a donc indissociabilité du processus et du produit qui convergent jusqu'à se superposer (ce n'est pas un hasard si l'improvisateur ne connaît pas à l'avance le résultat de ce qu'il jouera et ne le découvrira qu'au cours de son exécution musicale, alors que Beethoven, dans une lettre à Treitschke, exalte l'importance pour le compositeur d'avoir un regard global qui saisit et réfléchit l'œuvre dans son ensemble). $\mathrm{Si}$, pour celui qui compose, le temps possède une qualité de réversibilité (grâce à l'écriture musicale, il a la possibilité de revenir sur un passage, de le modifier ou de le corriger), l'improvisation en revanche a un caractère inéluctable, car tout passage joué même s'il n'est pas très réussi est définitif, fait immédiatement partie de la musique. En ce qui concerne le compositeur, qui a tout son temps pour décider ce qu'il veut " exprimer ", le processus d'exécution a moins d'importance, c'est le texte produit qui est important (au regard d'un jugement de valeur), le texte élaboré et raffiné ${ }^{7}$ La " composition " renvoie désormais au produit crée (la partition ellemême devient une chose en soi), alors que l'exécution apparaît comme auxiliaire. Précisément parce l'exécution ne coïncide pas avec un produit « externe » (elle a un résultat, mais pas de produit, excepté s'il y a enregistrement d'un disque), l'improvisation est au contraire constamment productive et expose sa propre pratique et le processus en cours. Comme acte unique, qui ne se répète pas et dont seul existe le " son », l'improvisation s'élide au cours de sa manifestation, et c'est précisément cette " dégradabilité ", considérée comme une menace à une époque où la musique propose de se libérer de sa nature temporelle, qui explique la marginalisation progressive de l'improvisation à partir de la fin du XVIII ${ }^{e}$ siècle ${ }^{8}$.

8 Étant donné l'époque historique et le cadre culturel du refus du « transitoire », il a fallu établir un système de notation capable de diviser les continus de la hauteur des sons et le rythme et ce de façon discrète et identifiable. L'écriture musicale n'est donc pas seulement un système codifié de marqueurs visuels (à savoir un algorithme qui produit des solutions efficaces au problème de la transmission du son et de la voix, fournissant un ensemble d'indications codifiées pour la conversion des signes en sons), mais aussi une stratégie pour " arrêter le temps » et mettre la musique en ordre des objets plutôt que des événements. Dans le sillage de la culture écrite et passant par des réseaux de transmission académiques, la notation ne permet pas seulement à la musique de devenir un document historique transmissible à la postérité, mais elle définit aussi l'œuvre, ses limites et la catégorie des exécutions fidèles à la partition ${ }^{9}$ en établissant, au moins de manière implicite, un idéal de normes auquel se conformer. La conséquence d'une telle révolution sera la conviction que la compréhension et l'appréciation des pratiques musicales performatives passent par le biais du concept 
d'œuvres autonomes et transcrites sur papier, négligeant, faute d'outils d'analyse, les autres musiques, en particulier les musiques des mondes non-occidentaux.

Mais comment ce changement s'est-il produit et quelles en ont été les conséquences?

10 Tandis que la notation est l'articulation spatiale et visuelle des sonorités, l'écriture est au contraire l'inscription du son dans un dispositif qui permet de "voir ", de décontextualiser et de dé-somatiser le son lui-même. Alors que l'écriture phonétique calque la prononciation, les grammata ne sont que des éléments abstraits. En principe, une œuvre sur partition n'a pas besoin d'être exécutée, puisque sa logique interne est parfaitement représentée par la notation, de même en est-il du livre qu'il n'est pas nécessaire de lire à voix haute pour en comprendre le sens. La concrétisation d'une œuvre peut au contraire être considérée comme une forme de corruption : «Why can't music go out in the same way it goes into a man, without having to crawl over a fence of sounds, thoraxes, catguts, wire, wood and brass. " ${ }^{10}$

11 Cette remarque de Charles Ives est révélatrice, car elle souligne à quel point la primauté abstraite et vidéocentriste de l'œuvre qui est créée, et ensuite fixée par la notation, inscrit l'acoustique dans une catégorie desonorisée, dévalorisant en même temps la fonction performative et la matérialité du son. Theodor Adorno s'exprime de manière assez semblable dans sa Théorie esthétique ${ }^{11}$, en regrettant presque le résonnement de la musique il affirme que les exécutions d'une oeuvre musicale sont moins intéressantes de sa partition et que celle-ci, à son tour, est moins intéressante de l'idée initiale du compositeur. Dans son Introduction à la sociologie de la musique ${ }^{12}$, il revient sur ce thème lorsqu'il fait l'éloge de la lecture « muette » qu'il définit comme la capacité à saisir l'essence vraie de la musique en faisant sien le texte musical grâce au « silence ». Citant Walter J. Ong ${ }^{13}$, un schéma alphabétique-textuel et rétinien a remplacé le paradigme oral/auratiquedes origines.

\section{Corps sonores}

D'ailleurs ce processus a une autre conséquence : il conduit à éluder le son en tant que produit d'une source incarnée. Un quatuor à cordes qui exécute un morceau de Mozart tend à produire des timbres tellement homologues qu'il devient difficile de les distinguer clairement. Comme s'il existait une tension à devoir dépasser le côté " physique » de la musique, comme s'il fallait se protéger de la présence du corps vivant de la musique en acte $^{14}$. Il ne faut pas oublier que la matrice de la musique est constituée de sons produits par des corps animés (ou par des corps non animés mais qui résonnent, qui sont agis pardes corps animés).

13 Dans la musique de jazz, par exemple, il suffit des premières notes d'un saxophone ténor (le contact particulier que le joueur établit avec son instrument au moment qui précède la production du son) pour savoir de qui il s'agit : Coleman Hawkins, Lester Young, Sonny Rollins ou John Coltrane. Produire des sons révèle un style propre à chaque musicien d'utiliser, de bouger et de faire vibrer son propre corps ${ }^{15}$. Il ne s'agit pas de jouer de l'instrument mais de faire résonner, par le biais de cet instrument, son propre corps ${ }^{16}$. Surgit alors ce que je nommerai une complicité ontologique entre corps et instrument. Le corps du musicien se confond avec l'instrument dont la proximité, l'intimité même, favorisent la fusion au point que le musicien n'a plus conscience de soi, de jouer un instrument ; la frontière entre individu et instrument disparait, et l'instrument est oblitéré de la représentation cognitive. À l'opposé, celui qui compose 
avec une plume n'est pas à l'intérieur de la musique, mais la voit comme un langageobjet à projeter ${ }^{17}$. J'insiste sur ce point : le jazz est une musique qui coïncide avec celui qui la joue, au sens littéral c'est le musicien qui « donne corps » à la musique. Il ne s'agit pas d'une pure spectacularisation. De fait, le musicien de jazz est « à l'intérieur » de la genèse de sa musique, il n'est qu'un avec elle, d'abord en tant que support matériel d'un processus où il est difficile de séparer l'auteur de l'œuvre-événement. Le corps de ses possibilités expressives coïncide avec les possibilités expressives de son corps. À la lumière de son expérience d'improvisation avec AAM, Cardew dit : «We are searching for sounds and for the response that attach to them, rather than thinking them up, preparing them and producing them $\aleph^{18}$. Le musicien ne produit pas des notes en ayant une conscience aiguë de ce qu'il est train de faire, comme si le son était quelque chose d'étranger à son corps, mais il doit être ce son. Ce qui compte ce n'est pas tant la mélodie ou l'harmonie mais le travail expressif $d u$ corps et sur le corps pour produire des sons qui vont au-delà de l'horizon de la note. Par contre, au cours d'un concert de musique avec partition, nous constatons un abandon progressif de la corporeité comme moyen d'expression privilégié, aussi bien de la part du musicien que de la part du public $^{19}$.

14 Un tel abandon est d'ailleurs lié à une surestimation de l'élément formel de la musique, de la syntaxe mélodique à la standardisation du timbre (l'enveloppe « bruyante » de la musique, quatrième paramètre avec la mélodie, l'harmonie et le rythme ${ }^{20}$ en passant par l'emphase sur la cohérence de l'œuvre, critères qui ne s'appliquent pas aux musiques non écrites ou non basées sur l'écriture en tant que dispositif de création. Dressé à se conformer à un modèle pré-écrit, ou à faire en sorte qu'il y ait un écart minime entre ce que la partition autorise et sa propre expression musicale, le musicien classique est entièrement au service de la partition, et l'introduction de quelque élément exogène, de quelque trace d'expérience ou de présence sensible de la part de celui qui la joue (inscrivant la musique dans le corps, dans la scansion érotique du joueur) est cachée. Ou alors condamnée : on est immédiatement accusé d'avoir une prise et/ou une position incorrecte où un doigté peu orthodoxe; de produire des notes pas nettes, de violer au fond le code imposé par l'institution pédagogique. Par contre, afin d'exploiter les possibilités expressives de leur instrument à vent plusieurs joueurs de jazz travaillent activement sur le bec : ralentissant, serrant, " mâchant " hanche et embouchure, c'est-à-dire faisant ce que l'enseignement du conservatoire aujourd'hui déconseille aux jeunes saxophonistes - ce qui explique aussi partiellement le petit nombre de musiciens ayant un son personnel. À une époque où l'élément visuel et logico-linguistique est exacerbé, de telles pratiques sont dépréciées esthétiquement, considérées non comme des différences d'expression capables d'amener à s'impliquer dans la musique, mais comme des sonorités ratées, « aberrantes " même. De sons qui attestent la présence vivante de celui qui les émet, on remarque plutôt les défauts et l'imprécision, le manque de détermination. Mais, prenons garde, de telles coloratures peuvent être considérées sans valeur d'échelle (donc une " dérive ") à condition que l'on assume comme référence obligée le schéma chromatique de la tradition européenne (une échelle de sons constituant une entité unifiée par l'octave), un standard d'intonation qui à son tour n'est pas problématisé. Il n'est pas seulement question du fait que le royaume des hauteurs est tout petit par rapport au monde des sons (rappelons-nous que le saxophone et la voix génèrent une gamme de sons qui ne coïncident pas toujours ni nécessairement avec la segmentation sonore - l'organisation par paliers d'un nombre de sons donnés - imposée par le système musical tonal ${ }^{21}$. Le 
point est que l'échelle tempérée est ab origine un compromis historique, comme le montre n'importe quelle étude anthropologique sur les systèmes d'intonation ${ }^{22}$.

\section{Contre l'austérité sonore : les expériences eurologiques}

C'est précisément en opposition à un tel horizon culturel que voient le jour, au cours du XXe siècle, trois nouveautés remarquables. La première est liée à la tentative de s'affranchir de la conception de l'écriture comme simple notation. La notation sur le pentagramme est remplacée par des graphiques faits de lignes et de points (et d'une série d'instructions qui indiquent précisément à l'exécutant quelles décisions prendre, comme dans un projet d'architecture). Parmi ces notations alternatives, il y a les diagrammes inventés par John Cage, ceux proposés par Earl Brown, sans oublier ceux qu'a élaborés Mauricio Kagel. Cornelius Cardew, à son tour, transforme la notation en un système sophistiqué de graphèmes (Treatise) ou en un ensemble d'instructions verbales (The Great Learning) ${ }^{23}$. L'exécutant se trouve ainsi face à des signes ambigus qui ne renvoient pas à un code sémantiquement univoque et qui, pour leur compréhension, nécessitent des explications supplémentaires. Ainsi au binôme traditionnel son/ton auquel chaque note est fixée, se substituent des symboles plurivoques qui suggèrent un ensemble de gestes et un domaine d'action.

La deuxième nouveauté concerne la valorisation du processus expérimental et donc la légitimation de l'œuvre ouverte qui prend la place de l'œuvre achevée, stable et définitive (L'Euvre ouverte d'Umberto Eco s'élabore précisément dans le contexte du Laboratoire de phonologie de Milan, grâce à la relation d'Eco avec Stockhausen et Luciano Berio). Dans Klavierstuck XI (1957) de Stockhausen, l'interprète a différentes options pour déterminer le rythme et l'intensité, et il peut, suivant son choix, exécuter les morceaux de musique sans respecter l'ordre chronologique, il peut aussi choisir de jouer ou partiellement ou intégralement l'œuvre, ou même rejouer un morceau, et l'œuvre ne prend fin que lorsque le même fragment a été joué pour la troisième fois ${ }^{24}$.

17 La troisième nouveauté et, pour nous, la plus remarquable a trait au refus de l'austérité sonore. En fait, l'histoire récente de l'avant-garde musicale du XXe siècle " peut être écrite comme la recherche ininterrompue de nouveaux timbres et de nouvelles sonorités ». Une recherche qui est commune à la musique d'avant-garde et à la musique populaire, du jazz à la techno, et qui constitue aujourd'hui l'une des voies les plus fécondes de la création musicale ${ }^{25}$. Recherche commune, certes, mais qui n'efface pas leurs différences majeures, comme nous allons le voir.

L'expansion du champ de pertinence du timbre à l'intérieur de l'événement sonore débute avec Alban Berg, Arnold Schoenberg et Anton Webern et leur choix de décrocher les notes de la référence privilégiée et " fonctionnelle » à la tonique (par exemple, les Cinq morceaux pour orchestre, op. 10, de Webern [1913]). Si déjà Schönberg, en déclarant que « les cas de notes qui sonnent mal n'existe pas [...], qu'il n'y a pas de limites aux possibilités de l'harmonie ${ }^{26}$, révélait sa volonté de repousser sans cesse la frontière tonale qui, dans le passé, limitait le territoire musical ; Edgar Varèse, quant à lui, se propose explicitement de créer des timbres jamais entendus auparavant : " La musique du futur ? Elle sera certainement basée sur le son et se situera au-delà des notes. [...] Notre alphabet musical doit s'enrichir $»^{27}$. Amériques (1918-1921), Hyperprism 
(1922-1923) et Ionisation (1929-1931) ont été conçus afin d'étendre et de renouveler le matériel sonore, en recherchant de nouvelles sources qui permettent de s'éloigner des hauteurs de la gamme chromatique (" lame stupide qui affecte l'octave », comme la définit Varèse). En 1940 Varèse déclare encore ouvertement qu'il préfère utiliser l'expression "son organisé ", au lieu de musique (terme, à son avis, inapte à définir toutes les expressions et les possibilités musicales).

Le refus de dépendre de la partition écrite, l'expérimentation avec des sons qui renient les organisations croissantes, la fusion de l'événement sonore avec le geste, d'où découle l'impossibilité de répéter la performance, la conviction que la totalité des sons auxquels ont peut avoir recours est potentiellement musicale sont les éléments que l'on retrouve aussi dans la musique de John Cage. La composante fondamentale de la proposition de John Cage est le postulat de l'équivalence de tous les phénomènes sonores. Déjà dans The Future of Music (1937), Cage légitime l'utilisation à des fins musicales de «tout son qui peut être entendu ». L'idée étant que le son a une valeur en soi, indépendamment de sa position dans une succession. En ce sens, il n'y a pas mélodie mais émergence de sons-événements complets en soi et donc sans nécessité d'un " développement » au-delà de leur coexistence ou succession dans l'espace. En 1957, dans Experimental Music, Cage déclare que ce qui l'intéresse c'est n'importe quel nombre de sons, dans n'importe quelle combinaison et avec n'importe quelle continuité. À ce sujet, 4'33" représente un cas emblématique : cette œuvre consiste uniquement en une pause partagée en trois mouvements, ou mieux, en trois tacet différenciables par le geste (cette œuvre fut " exécutée " pour la première fois par David Tudor le 29 août 1952 à Woodstock). L'auditeur est laissé seul face à l'aléa sonore de l'ambiance - à la phonosphère, aux sons qui jaillissent partout autour de lui ${ }^{28}$. Dans " 4.33 ", la seule donnée qui reste est sa composante unique, commune au son et au silence : le temps.

\section{Contre l'austérité sonore : le jazz}

Même dans le jazz - surtout dans le jazz des années 1960 - la recherche de la nouveauté prend la forme d'un refus de la référence thématique et de la trame harmonique en tant que passages obligés pour celui qui joue et cette recherche se veut par-dessus tout une exploration inconditionnée de l'univers sonore, exploration des sons sans hauteur définie, des tessitures sonores, des sonorités considérées comme parasites, des sonorités étrangères aux cultures occidentales. Bref, elle a recours aux " ultrasons " (ainsi pourrait-on les nommer) que l'on écoute non pour saisir une succession de notes mais pour se laisser surprendre par l'impact phonique. Selon Ornette Coleman, «most western music originates with the piano, so that limits musicians to the diatonicchromatic system and inhibits the instrumentalists. I want a more human expression that's not limited to tempered notes $»^{29}$.

21 "We don't need notes anymore ", remarque à son tour le saxophoniste alto et compositeur Anthony Braxton, affirmant que l'un des aspects les plus significatifs de l'improvisation dans le jazz, est sa capacité à dépasser l'horizon de la note et d'accéder à l'« anotalité » : «I'm looking for instruments that are not concerned with actual fixed pitches, instruments with whirls of sound in them ". "Sound - not pitch - that's the difference ", ajoute enfin Roscoe Mitchell, membre de l'AACM (Association for the Advancement of Creative Musicians), fondée dans le quartier de Chicago, the all black 
South Side, en 1965 (Sound est aussi le titre d'un célèbre disque enregistré par R. Mitchell en 1966 pour la maison de disques Delmark).

Or, par rapport à la proposition de Cage, un élément de différence crucial est introduit : de tels sons deviennent des vecteurs prégnants d'un projet qui met en gros plan l'idée du corporel. Un exemple intéressant qui illustre cette notion du corps-instrument ou soundful body se trouve dans des morceaux comme "You did it, you did it » et « Black root » du multi-instrumentiste Rahsaan Roland Kirk (respectivement dans les disques We free kings, Mercury, 1961 et dans Natural Black Inventions : Roots Strata, Atlantic, 1971). Dans ces morceaux, Kirk joue de la flûte avec une technique où il souffle et vocalise en même temps. Voix, souffle et instrument sont une chose, et nous, qui écoutons, découvrons un espace intermédiaire où ce qui résonne est le corps. Ce qui frappe, c'est la technique du dual voicing où l'on souffle et vocalise (ou on chante ou parle) en même temps, produisant des split tones ou multisons (c'est-à-dire l'émission simultanée de sonorités dont les différentes fréquences deviennent perceptibles), mais c'est surtout la dimension charnelle du son. Des phrases rauques, des rots sonores, des souffles, des grognements, des hurlements, des gémissements, des couinements, des effets de timbres, des humming font partie de la performance, ils manifestent la présence d'un corps sonore et révèlent une sorte de performatif biologique (une prétention à la vie) où interagissent, entre les deux axes de l'expressivité, l'axe vertical ou biologique et l'axe horizontal ou culturel. Il s'agit parfois de sons peu musicaux, mais comme l'a raconté une fois Picasso à Gertrude $\operatorname{Stein}^{30}$, ceux qui créent quelque chose pour la première fois sont obligés de la rendre laide, étant donné l'effort et la lutte nécessaires à son engendrement. Monk parlait à ce propos d'une « beauté horrible » (ugly beauty). Ceux qui suivront pourront, en revanche, la perfectionner et la rendre belle parce qu'ils sauront ce qu'ils sont en train de faire, étant donné que la catégorie ou la technique ou le style ont déjà été inventés.

23 Si le thème central de la linguistique est le son qui devient signification, ce que nous faisons ici c'est de retracer la formation du sens au seuil même de son émergence sensible et incorporée. Écouter l'improvisation de Kirk, c'est être projeté au sein de quelque chose qui caresse et qui jouit, qui racle et qui souffre. Le musicien offre son propre corps comme surface de résonance qui se tend et se détend, se gonfle et se dégonfle, qui est pincé, frappé, frotté. On découvre que la musique - parce que produite par un corps dans lequel coexistent processus pulsionnels et fonctions vitales - a un statut non seulement esthétique mais aussi charnel. Le souffle d'air - considéré comme importun par la musicologie (et donc comme quelque chose devant être supprimé) est, par exemple, parfaitement audible dans le timbre de Kirk ${ }^{31}$. Il traverse des organes du corps destinés au maintien de la vie et en assume le rythme et la sonorité, l'entière présence d'un corps humain est ainsi exprimée (littéralement "pressée au dehors »). Les organes ne sont pas silencieux (comme un corps vidé le serait) et c'est ce bruissement du corps qui est en jeu dans les gestes sonores du jazz ${ }^{32}$.

24 Là où, dans le domaine de la soi-disant musique classique, la présence du corps a été disciplinée de façon telle qu'elle est imperceptible, dans le contexte afro-diasporique le corps du " performer » devient une audible présence. Selon Stuart Hall, les membres de la diaspora noire - marginalisés par un monde logocentrique dont l'accès et la maitrise des codes culturels coïncident avec la maîtrise de l'écriture - ont trouvé la forme et la structure profondes de leur vie culturelle dans la musique. Ils ont utilisé leur corps comme s'il était, et souvent il l'a été réellement, leur seul capital culturel et ont 
travaillé sur eux-mêmes comme s'ils étaient le matériel même de la représentation ${ }^{33}$. Nous ne devons pas oublier qu'il n'a été concédé aux Afro-Américains que de minimes espaces de reproduction de leur propre identité. La musique en est venue à représenter l'un de ces contextes (esthético-expressifs) où ils pouvaient non seulement se réunir dans des situations de coprésence mais aussi se présenter comme des personnes capables de créer. Mais la portée anthropologique du jazz n'interpelle pas seulement un domaine d'excellence humaine enraciné dans l'action d'invention, elle permet aussi de s'opposer à la dé-singularisation, c'est-à-dire à la tendance raciste à traiter chaque individu comme specimen d'un type (l'adjectif « noir » fait référence à une classe entière d'individus considérés comme équivalents, donc interchangeables), selon un processus inverse de celui qui nous différencie et nous rend singuliers. Et c'est précisément grâce au timbre particulier de chacun que se révèle sa singularité incarnée ${ }^{34}$. En tant que prolongation du musicien, la sonorité devient alors l'équivalent de la différence expressive entre chaque individu.

Pour revenir à l'improvisation de Kirk, ce qui est palpable ce n'est pas la " seule » musique, mais ce que Barthes appelle des somathemes ${ }^{35}$, les gestes du corps dans leur conduite musicale. C'est peut-être pour cette raison que Barthes dans Le grain de la voix parvient à distinguer dans le vocalique une catégorie grâce à laquelle on pourrait écrire une contre-histoire de la musique, une histoire où une interprétation ne serait pas jugée à partir de critères de style - rigueur, éclat, fidélité à la partition - mais par rapport au corps et à la restitution de sa présence au travers de la musique.

Je ne voudrais pas qu'il y ait un malentendu à ce sujet. L'attrait de la "nudité » du vocalique ne doit pas nous pousser à considérer de tels gestes sonores et performatifs comme une expression du corps immédiate, ou même comme un indice de la proximité à la nature (donc comme un signe d'infériorité cognitive). Ce n'est pas seulement le corps du concertiste qui est contrôlé, mais aussi celui du musicien de jazz : il s'agit dans les deux cas de corps disciplinés mais de manières différentes. Autrement on retombe dans la mythologie du corps - celui du joueur de jazz - entièrement libre, là où le corps est une sorte de carrefour de stratégies de domination (discipline), mais aussi d'affranchissement de la discipline. La conviction selon laquelle l'improvisation implique une absence de discipline et de technique instrumentale n'est que pure idéologie, souvent construite par la partie adverse des Blancs ${ }^{36}$. La compétence de celui qui improvise est le résultat extrême d'un long apprentissage. Charlie Parker, par exemple, ne s'est pas distingué comme génie musical dès sa naissance. Considéré comme un dilettante au son exécrable, il a subi des humiliations. Gene Ramey, qui a joué avec Parker à Kansas City, se souvient de lui comme d'un obsessionnel : il avait appris par cœur tous les solos de Lester Young et lorsqu'ils eurent l'occasion de jouer ensemble en 1946 pour « Jazz at the Philarmonic »de Norman Granz, on entend Parker in Lady be good citant explicitement certaines phrases improvisées par Young dans un enregistrement du même morceau. Comme le disait aussi Miles Davis : "You don't practice, you can't play nothing $»^{37}$, et il ajoutait qu'il lui avait fallu deux ans de travail acharné pour retrouver son propre ton (ou, en tout cas, un ton qui lui plaisait) après une absence temporaire de la scène musicale.

Le parcours pour se former était en fait long, complexe et absorbant, et il comprenait de pratiquer avec l'orchestre de l'école, dans les jam sessions informelles, il y avait aussi l'enseignement à la maison (home schooling) et l'apprentissage autodidacte. Comme dans certaines pratiques orientales où l'élève s'installe dans la famille du 
maître, ainsi en était-il dans la communauté noire : celui qui avait sérieusement l'intention de faire du jazz (même s'il n'était pas noir, comme Paul Bley) était " adopté " par les cercles attitrés. Il ne faut surtout pas oublier qu'à cette époque, il était impossible de télécharger les transcriptions de milliers de solos improvisés. Il fallait donc pratiquer seul : écouter, répéter, et répéter encore ${ }^{38}$.

Comme le souligne Cecil Taylor, la physicité de la musique afro-américaine est fondamentale : « Horace [Silver] has a certain kind of physical movement, a certain way he attacks the piano. If you notice Daddy Ellington, when he plays you'll see that he has a certain thing that he does with his foot, and that foot action is as much part of what's happening with the keybord as his hand $»^{39}$. Précisément parce qu'elle est provoquée par le mouvement du corps, la musique produit à son tour des réactions sur le spectateur. C'est grâce à la présence vivante et é-vocatrice d'un corps sonore que je suis affecté. Le pouvoir phatique d'établir un contact et de susciter des effets sur un auditoire de réception, c'est ce qu'Austin définissait comme la force de l'acte performatif. Revenons à Cecil Taylor et à la manière dont il se sert du clavier comme d'un territoire de registres sonores dont il utilise les touches pour la densité de la résonance, alors on comprend que Taylor produit beaucoup d'air, littéralement des ondes sonores qui ont un impact sur la peau, sur le torse, sur les os de l'auditoire, qui a l'impression d'être massé-manipulé par le son.

En outre, la musique n'existe que si des corps donnés la produisent par un certain effort physique. Produire des sons requiert de la force, de la coordination et de la souplesse. « There is no music than can be performed unless somebody plays it» $»^{40}$ note Ornette Coleman. Et Jeanne Phillips, une afro-américaine spécialiste de jazz lui fait écho : « All these cats with their electronic stuff - how the hell is a machine going to make music? No more than a washing machine does when its operating. You hear sounds all day long, and unless they're incorporated correctly by men, there ain't no music $»^{41}$. Cecil Taylor remarque à son tour, «David Tudor is supposed to be the great pianist of the modern Western music because he's so detached.You're damned right he's detached. He's so detached he ain't even there. [...] The body is in no way supposed to get involved in [the music] $»^{42}$

\section{Le corps manqué : sur la différence entre la tradition afrologique et eurologique}

Même si Cage et le jazz ont en commun l'expérimentation avec les sons et la production d'une musique imprévisible, la différence majeure entre eux est que le jazz ne se veut pas le fruit du hasard (ni de sa cécité, au contraire de Cage qui, lorsqu'il compose, recherche le moyen de n'avoir aucune connaissance de ce qui pourrait arriver ${ }^{43}$ ), mais d'un télescopage entre hasard et contrôle. Le musicien de jazz s'exerce activement à cette habilité qui consiste à " exploiter " l'imprévisible en l'absorbant pour y saisir le vecteur d'actions musicales à venir. Mais Cage n'est pas en quête d'un moment de serendipity, la fortuite mais agréable occurrence de quelque chose qui facilite le développement de la musique. Dans le jazz, la musique se déploie dans des directions que personne n'est en mesure de prévoir, alors même que c'est précisément cette même musique qui va donner l'occasion au musicien de révéler sa capacité d'action. Dans quel sens pouvons-nous assumer la responsabilité d'une musique - et l'accepter comme " notre »- si elle a lieu d'une façon parfaitement aléatoire? Si la logique du 
développement d'un solo improvisé ne rassemble pas à une route rectiligne, elle ne ressemble pas non plus à un processus stochastique. La performance de Cage, comme les performances du jazz sont par principe ouvertes. Il y a toutefois une différence subtile mais déterminante entre le non-intentionnel et le non-contrôlé (mais parfaitement préparé) de Cage, et c'est le fruit imprévisible, excédant tout projet prémédité et pourtant parsemé de moments d'une intervention active et " conductible ", propre au jazz. C'est un peu comme un skipper qui pour naviguer en pleine mer dépend des courants mais cherche aussi à les exploiter de façon à pouvoir conduire ou au moins rapprocher son bateau d'une route. Ce qui est important c'est le télescopage (interplay) entre les forces naturelles, le hasard et le contrôle.

Tandis que le compositeur aléatoire est « auteur » d'une musique qui, à la rigueur, n'est pas la sienne, le musicien de jazz « travaille » principalement sur des sons produits intentionnellement par des êtres humains avec un instrument, sur des sons, en principe, modulables. Cage, nous l'avons vu, n'est intéressé ni par les sons émis par un instrument musical ni par les sons produits par la voix humaine, mais par la sphère acoustique audible en tant que contexte global de sons, quelque soit leur origine : « les sons ont toujours lieu, qu'on les produise ou pas $»^{44}$. Un tel refus de la part de Cage est nourri par le désir de dépouiller la musique de la présence de dispositions et inclinaisons personnelles ou culturelles (de la nécessité de soumettre tout choix musical à des calculs arbitraires qui la rendent ainsi indéterminée). Dans la méthode de composition de Cage, ce qui arrivera est déterminé par le recours aux lois de la probabilité (par exemple, un coup de dès) ou par la sélection d'un mécanisme de génération automatique destiné, une fois programmé, à établir la position et la combinaison des notes. Renonçant à son propre désir de " contrôler " le son, Cage insiste dans Silence à ce que le compositeur se limite à préparer les matériaux de base et à donner des indications (avec beaucoup de soin, même parfois excessif si l'on pense au nombre de grilles, de tableaux, de schémas) sur les conditions initiales d'une performance dont le déroulement sera indéterminé. L'inattendu a ainsi lieu par nécessité et, en tout cas, abstraction faite de l'interaction entre les musiciens (et une structure de probabilité ne peut être comparée à une improvisation de groupe). Nous avons affaire à une musique "induite » et autopoïétique qui n'est pas générée par des régimes d'interaction, une musique qui n'interpelle pas le monde du corps à corps vu dans le jazz, mais une sorte de laboratoire scientifique. Par la promotion d'expériences qui « permettent aux sons d'être eux-mêmes, eux-mêmes plutôt que des véhicules de conceptions ou expression d'attitudes déterminées ${ }^{45}$, le son musical devient simplement une forme de résonance ambiante. Une fois ramené à l'ordre pur du sonore, le musical perd son côté humain, son lien intégral avec sa matrix qu'est le corps sonore. Les performances de Cage, séparées de leur source humaine, sont alors embedded dans un contexte, mais rarement embodied. En ce sens Cage a "manqué le corps » (ou a peut-être a-t-il tenté d'en évacuer la matérialité) ${ }^{46}$.

32 En proposant mes observation sur la musique en tant qu'activité (et non pas en tant que produit) où participent des corps sonores, et même si j'ai soulevé la question du destin des pratiques orales dans les cultures de la représentation et de l'image, mon intention n'était pas de faire une lecture antagoniste, de remettre en cause l'écriture, d'un côté, et de revaloriser la dimension corporelle du son, de l'autre. Il ne s'agissait pas de révéler une nostalgie inavouée - ou même une célébration - de l'oralité ${ }^{47}$, mais plutôt de montrer que les paradigmes d'évaluation des musiques centrées sur 
l'improvisation ont émergé dans le contexte d'analyse de la musique notée et sont donc structurellement inadéquats à rendre compte de ces musiques.

Je ne voulais pas non plus établir une classification des différents types d'identité sonore (l'émission de bruits de la part de l'humain, de l'animal, les gestes humains liés aux instruments musicaux, sources mécaniques, sons ambiants), ni même considérer si l'approche textocentriste conduira à l'apprivoisement de la pratique de l'improvisation, mais je voulais avant tout réfléchir à la façon dont le signe écrit a été pensé dans la musique, au type de sujet auquel il renvoie et aux conséquences d'un tel tournant sur l'écriture.

J'ai montré jusqu'à quel point la musique en Occident s'est développée sous le contrôle progressif de l'oreille de la part de l'œil, avec l'adoption d'un paradigme « oculaire », en passant de la " mise en résonance » à la " mise en évidence $»^{48}$, ou mieux l'é-vidence, comme l'appelle Heidegger ${ }^{49}$. Le son devient connaissable et compréhensible à partir du moment où il est décomposé, sectionné en segments, en coupant le flux sonore médian au moyen d'une grille, de façon à pouvoir voir, compter et conserver les parties. Les sons, invisibles par définition, deviennent visibles en formes de points situés sur une grille à intervalles (des lignes qui coupent la feuille horizontalement). Mais le vidéocentrisme ne se réfère pas seulement à un effet technique et musical, ni ne renvoie non plus au regard de chaque individu, mais il interpelle en revanche le contexte culturel qui définit la place du voir en tant que système de représentation (selon Heidegger, le trait fondamental de la modernité est le fait que le monde s'est résolu « dans une image $\aleph^{50}$ ). Un tel vidéocentrisme a produit un excès de graphocentrisme en musique dont les conséquences ne sont pas du tout inoffensives : dans le contexte du jazz, par exemple, on privilégie de façon unilatérale les morceaux transcrits (de Duke Ellington, par exemple), les enregistrements « lisses » d'un Stan Kenton et l'improvisation caractérisée par la reproduction " propre » des notes plutôt que la manipulation du timbre et l'exploration de possibilités rythmiques. La production de notes dans des champs considérés comme peu " pertinents " par rapport à la motivation thématique résulte incohérente, pâles imitations du monument à la beauté - à la perfection - offert par la musique composée et raffinée. En conclusion, un ordre esthétique s'est affirmé qui idéalise la musique dans sa détermination objective au détriment aussi bien du processus performatif que de la qualité individuelle et individuante du son, et enfin des sonorités inhabituelles. La distinction entre son et note se transforme dans une opposition, même plus, une subordination hiérarchique du premier vis-à-vis de la seconde - comme si la caractérisation du timbre du son était reconduite à l'indétermination sémantique et donc à la confusion. Mais c'est justement un tel ordre esthétique, fondé sur le signe écrit, à révéler le paradoxe qu'il cache en soi : en tant que tentative de fixer la musique à l'intérieur d'un système de représentation stable, contrôlable et reproductible, la notation est une reconnaissance implicite du fait que la musique est insaisissable et difficile à retenir. Étant donné qu'elle est une déclaration contre l'improvisation et les sonorités non codifiées, l'indéterminé sera présent derrière chaque acte d'écriture musicale.

J'ai tenté ici de montrer comment, grâce à Cage et au jazz, la distinction entre signal et bruit redevient floue. Plus, c'est au statut même du bruit de changer : il n'est pas quelque chose de négatif à réduire ou à supprimer - en supprimant avec lui la contingence, l'imprévisibilité et l'exceptionnalité -, mais une ressource potentielle à laquelle il ne faut pas renoncer pour extraire de nouvelles formes de sens musical. Cela 
dit, j'ai voulu souligner comment Cage et le jazz, avec deux attitudes entièrement différentes, ont rendu instable la séparation entre le musical et le sonore. Le jazz exalte le rôle du corps en tant que condition de possibilité matérielle de la musique. Et si un tel élément a été supprimé dans la musique soit disant sérieuse ou contemporaine, c'est, je crois, dû au fait que le son non lexicalisé émis par une source charnelle (appelons-le " vocalique ») fait peur, effraie parce qu'il est le phénomène qui précède l'articulation verbale et la segmentation tonale, un phénomène que l'on confond avec la dissonance et l'indistinction, lesquelles constituent, à leur tour, le refoulé sur lequel se fonde l'ordre du logos.

C'est précisément cet aspect corporel que le jazz ne cesse d'évoquer.

\section{Annexe}

Dans mon exposé je ne voulais pas affirmer que les échanges entre les traditions afrologique et eurologique étaient inexistants. Les échanges au contraire sont fréquents (même Charles Ives a enregistré en privé une petite série de fragments improvisés entre 1938 et 1943) mais ont étés souvent cachés ou présentés de façon tendancieuse comme nous allons voir.

Voyons d'abord Edgar Varèse. Olivia Mattis ${ }^{51}$ a montré ce que le Poème électronique, crée pour le Pavillon Philips projeté par Le Corbusier pour l'Exposition Universelle de Bruxelles en 1958, doit à la rencontre/confrontation de Varèse avec la scène du jazz de New York des années 1950 (même à la marge de certaines mesures de Déserts l'on trouve la notation en lettres capitales «JAZZ » et il ne faut pas oublier que Varèse avait organisé une performance de Poème au Village Gate). Bien que voisin de Charlie Parker au Greenwich Village Varèse ne s'était jamais senti trop attiré par le jazz (Parker en revanche rendra visite à Varèse plusieurs fois et lui rendra ouvertement hommage). Toutefois, après la mort de Parker, Varèse commence à développer un intérêt pour le jazz et pour la liberté performative en général. Autant qu'en mars et en août de 1957, juste avant son départ pour l'Europe, collaborera avec des grands musiciens du jazz, comme le trompettiste Art Farmer, le saxophoniste ténor (et producteur de Miles Davis pour Columbia) Teo Macero et Charles Mingus. Earle Brown sera l'organisateur de l'événement qui aura lieu à la présence de John Cage et de Merce Cunningham. Les sessions consistent dans une alternance de pulsations rythmiques régulières, avec des moments où plusieurs musiciens convergent vers les agglomérats timbriques d'une masse sonore. Du point de vue du jazz elles ne constituent pas des nouveautés mais sont des expériences significatives pour Varèse qui arrivera en août 1957 avec une collection des disques de Mingus et la trace de la partition de la session de jazz. Varèse a toujours recyclé du matériel tiré des compositions précédentes et au Poème électronique intégrera différents éléments des sessions de jazz de 1957 (les éléments rythmiques et l'alternance sonore sont par exemple bien présents dans le Poème). De plus si l'on compare le brouillon de la partition du Poème et la partition, sans titre, tracée par Varèse pour les sessions improvisés, la ressemblance, même l'identité, est frappante (une photo de deux partitions est publiée dans l'article de Mattis).

Pour continuer sur les relations entre traditions eurologique et afrologique, Conlon Nancarrow a été trompettiste de jazz sans vouloir de ma part signifier que cette expérience n'ait constituée qu'une " parenthèse temporaire et de juvénile ». 
Harry Partch s'inspire au jazz non seulement du point de vue musical mais aussi du point de vue symbolique pour sa pièce de théâtre Water ! Water ! de 1961 où le jazz évoque le retour de la pluie après une longue période de forte sécheresse.

D’ailleurs Markus Stockhausen, fils de Karlheinz Stockhausen, est trompettiste de jazz.

Pour ce qui concerne le monde du jazz, sans aller jusqu'à citer le thrid stream (Brubeck étudie avec Milhaud, John Lewis intègre polytonalité et contrepoint dans le jazz), il ne faut pas oublier qu'après la seconde guerre mondiale Stockhausen fut séduit par les concerts européens de George Russell et qu'il cite le free jazz et la musique indienne comme influences fondamentales avant sa condamnation publique du jazz à la fin des années 1960. Grâce à Schuller, Dolphy exécute Density 21. 5 (1936) de Varèse. Braxton compose pour Merce Cunningham, joue en 1970 (comme Steve Lacy) avec la bande d'improvisation Musica Elettronica Viva et étudie avec Morton Feldman et Earl Brown : December 1952 en particulier influencera son mélange singulier de partition notée, instructions verbales et diagrammes. Joseph Jarman (en quintet) e John Cage collaborent en novembre 1965 à Chicago au cours d'une performance (critique musicale sur Down Beat ) : Cage produit des sons électroniques auxquels Jarman répond. Cage a partagé la scène avec Sun Ra au cours d'une lecture de leurs poèmes respectifs à New York en 1986.

3 Yoko Ono a interprété aussi bien la musique de Cage que collaboré avec Ornette Coleman, Aos en 1965, en improvisant des gémissements d'une femme au sommet de l'orgasme.

44 Une composition d'Ornette Coleman, City Minds and country Hearts a été exécutée en première mondiale par David Tudor et par la celliste Charlotte Moorman à New York en 1963.

4 Pour ce qui concerne la musique aléatoire, une performance de Roscoe Mitchell ayant pour titre Cards consistait en une série de feuilles de partition notées qui étaient mélangés au hasard et découvertes l'une après l'autre au cours de la performance.

6 Même Braxton qu'avec Leo Smith utilisait souvent au cours de ses performances seau, bâtons et pierres, une fois avait grimpé sur un escabeau et avait commencé à jeter des objets dans une poubelle.

Mais malgré tous ces exemples la relation entre le deux traditions a été relatée souvent de façon partielle et déformée. Par exemple, dans un entretien Cage fait référence à la performance avec Jarman à Chicago que nous venons d'évoquer en se présentant comme l'artiste d'avant-garde " donne des leçons » à un groupe de musiciens noirs non-identifiés (G. Lewis, A power stronger than itself. The AACM and American experimental music, University of Chicago Press, 2008).

même façon, lorsque Ornette Coleman enregistre son œuvre pour orchestre Skies of America, la production CBS la découpe en différents morceaux et leur donne des titres comme s'il s'agissait des chansons et non pas d'une symphonie. L'idée implicite est que les noirs ne sont pas autorisés à composer de la musique comme si le label « saxophoniste jazz " leur empêchaient de jouer la musique selon styles et modes différents.

49 Toujours définie comme « pure jazz » la contribution afro-américaine à l'expérimentation du XXe siècle est supprimée en tant que discours. Ce n'est pas un hasard si les compositeurs classiques afro-américains ont étés si largement ignorés même par les intellectuels de la culture afro-américaine. 


\section{NOTES}

1. Carl Dahlhaus, Was heißt Improvisation, in Improvisation und neue Musik : Acht Kongressreferate, ed. Reinhold Brinkmann, Mainz, B. Schott's Söhne.

2. Hans Jonas, "The Nobility of Sight. A Study in the Phenomenology of the Sense", Philosophical andPhenomenological Research, vol.14, 4, p.507-519.

3. Certes si l'objet est grand et/ou complexe, par exemple une cathédrale ou un jardin, cela demande du temps pour être saisi «entièrement » dans un battement de cils. Même une statue oblige le spectateur à se déplacer de façon à pouvoir apercevoir ses différentes propriétés dans une succession temporelle comme si la statue pouvait «se livrer » progressivement et non pas simultanément (cette circonstance paraît atténuer la grande répartition entre arts de l'espace et arts du temps).

4. «La musique donc qui naît et se consomme en même temps est moins digne de valeur de la musique ».

M. Garda, "La teoria dell'opera musicale del Novecento", in Storia dei concetti musicali. Espressione, forma, opera, Carocci, Roma, 2007, p.249-250.

5. «Mais la peinture excelle et domine la musique parce qu'elle ne meurt pas immédiatement après sa création, comme cette malheureuse musique, mais elle reste et montre comme vivant ce que, en réalité, n'est que surface. »

Id., L'estetica musicale del Novecento. Tendenze e problemi, Carocci, Roma, 2007, p.133

6. Vidéocentrisme est la référence lexicale que j'utilise pour indiquer la primauté du voir et du «visible» dans notre culture de représentation. Figures et métaphores optiques comme le « regard », le « spectateur » et le « public », qui incarne la totalité sociale en tant qu'observateur qui juge, sont des symptômes utiles de cette primauté. En faisant référence à une culture «typographique » McLuhan - Marshall McLuhan, Gutenberg Galaxy. The Making of typographic Man, Toronto, Buffalo, Londres, University of Toronto Press, 1962 - affirmait que l'alphabétisation «avait donné un œil à l'oreille » et avait ainsi instauré une sorte de primauté du voir sur les autres sens. Voir aussi R. Barbanti, «Crisi e persistenza del modello retinico occidentale. Elementi per la definizione di un nuovo paradigma acustic »o, in Musica e suoni dell'ambiente, edit. par A. Mayr, Bologne, Clueb, 2001 et Id., "Meccanicismo e determinismo. Ovvero come lo sguardo, fissandosi sulle cose, ha prodotto una visione del mondo riduttiva", in Ecologia della musica. Saggi sul paesaggio sonoro, edit. Par A. Colimberti, Rome, Donzelli, 2004, p. 79-99.

7. Dans ma distinction entre composition et improvisation je ne propose pas d'établir une hiérarchie, mais plutôt de mettre l'accent sur leurs qualités réciproques. J'utilise le terme qualité non pas en tant que valeur mais dans le sens originaire du terme, à savoir comme manière d'être propre à une chose.

8. Selon Cardew, "Towards an Ethics of Improvisation", in Id., Treatise Handbook, Edition Pçeters, Londres 1971, p.46: «Written compositions are fired off into the future; even if never performed, the writing remains a point of reference. Improvisation is in the present; its effects may live on the souls of the participants (...) but in its concrete form it is gone for ever from the moment the it occurs, nor did it have any previous existence before the moment that it occured, so neither is there any historical reference available » De nos jours la musique est aussi ou surtout le résultat d'une «médiation" et d'une reproduction; l'enregistrement est désormais une partie constitutive de l'événement musical et non pas quelque chose d'extérieur qui s'y ajoute. D'ailleurs même l'improvisation peut être transcrite et donc reproduite. Mais il ne faut pas confondre un morceau transcrit avec l'enregistrement d'un morceau improvisé. À la différence de la notation, l'enregistrement n'est pas «exécuté » en public. La partition a une valeur de norme et rend la musique répétable (de façon plus ou moins univoque) indéfiniment. En principe 
l'enregistrement (ou la transcription) d'une performance improvisée peut avoir cette valeur, mais cette dernière n'est qu'un souvenir, un dispositif d'apprentissage ou d'analyse, il n'a pas une valeur esthétique et celui qui prétend improviser à partir de l'exécution d'un solo bien transcrit d'un autre musicien serait condamné par la communauté des musiciens de jazz. C'est pour cela que l'enregistrement est par essence vide.

9. Nelson Goodman, Languages of Art: An Approach to a Theory of Symbols, Bobbs-Merrill, Indianapolis, 1968.

10. Charles Ives, Essay before a Sonata. The Majority and Other Writings, W. W. Norton ,New York, 1990.

11. Theodor W. Adorno, Théorie esthétique (1970), trad. Marc Jimenez,Klincksieck, Paris (1974) 1985.

12. Theodor W. Adorno, Introduction à la sociologie de la musique (1962), trad. Vincent Barras et Carlo Russi, Contrechamps, Paris, 1994.

13. Walter J. Ong, Orality and Literacy: The Technologizing of the Word, Routledge, London and New York, 1982

14. Comme Michel Foucault et Norbert Elias l'ont montré, à partir du XVIIe siècle le corps connaît une marginalisation progressive et une tendance au contrôle des « défoulements de la matérialité » commence à se répandre : au jour le jour l'expression directe du corps (de la naissance à la mort, de la folie à la sexualité) devient en public "dégoûtante ». La société convenable et sans odeurs est le dernier fruit d'une longue histoire sociale qui a fait du corps une honte à cacher. Même l'exercice du pouvoir se rationalise et se despectacularise (Foucault parle de «normalisation»): les exécutions capitales et les tortures publiques ne sont plus utilisées par l'état pour garantir l'ordre établi. La société ne devient pas plus humaine mais de telles méthodes sont désormais considérées comme incompatibles avec les nouvelles institutions médico-légales. En suivant cette forme de normalisation, les aspects liés au corps «non-discipliné » sont relégués dans les coulisses.

Pour revenir à la musique, il faut remarquer la tendance actuelle de la part de plusieurs écoles, sous l'influence des acquis de l'ethnomusicologie, à laisser la place au corps non seulement dans le chant mais aussi dans l'exécution de la musique, même baroque.

15. Ce n'est pas un hasard si parmi les aspects spécifiques qui qualifient l'approche au processus de la production de musique d'origine africaine olly Wilson cite «the tendency to incorporate physical body motion as an integral part of the music making process ", p.329 (Olly Wilson, « The heterogeneous sound ideal in african-american music " in New Perspectives on music: essays in honour of Eileen Southern, Warren, Harmonie, Park Press, 1992, p327-338). En revanche Samuel Floyd fait référence à une Ring Shout Aesthetic : la Ring Shout était à la fois de la musique, de la danse et la pratique religieuse caractérisée par «calls, cries, gollers, call-and-response devices, blue notes, ben notes, hums, moans, grunts, interjections and punctuations " p.6 (Samuel Floyd, The Power of black Music : interpreting its history from Africa to the United States, Oxford University Press, New York, 1995).

16. Pour montrer que la singularité du timbre est liée à l'inflexion du joueur et non pas à l'instrument (à savoir que le timbre ne peut être acheté dans un magasin avec l'instrument) il est intéressant de reporter le récit de Lee Konitz - sax alto - au cours d'une conversation. Un soir Charlie Parker n'a pas son instrument et l'emprunte à Konitz. Celui-ci est alors abasourdi par les sons que Parker tire de son instrument. Lorsque Parker le lui rend Konitz exprime son espoir de retrouver dans son instrument encore « un petit peu » de la musique époustouflante jouée par Parker (A.Hamilton, Lee Konitz. Conversations on the improviser's art, University of Michigan Press, Ann Arbor, 2006). Même avec un piano - qui est considéré comme un instrument structurellement incapable de produire des inflexions vocales - certains pianistes réussissent avec la pression de la main sur le clavier à en faire ressortir des sonorités entre les interstices des 
notes, à obtenir quelque chose de similaire à ces distorsions tonales que les instruments à vent produisent par le biais du timbre.

17. Cecil Taylor affirme: "Si vous donnez à votre physicité la tâche de lire les notes qui éventuellement sont là depuis 500 ans au lieu de créer votre propre système de notation, vous divisez la concentration de votre énergie ", (conférence publique à Palazzo dei Notai, Bologne, 11 mai 2000). Et encore « one of the distractions has been this idea of written music. It divides the sense, though some people might say it increases the options. But I mean if you look at a piece of music - notes - that means your eye must be directed outside the body» p.231 (H. Mandel, Miles, Ornette, Cecil. Jazz beyond jazz, Routledge, New York, 2007). Avec ces remarques Taylor met l'accent sur les rôles différents de la mémoire: incorporée, d'une part, délocalisée dans les textes, y compris dans les partitions, de l'autre. Pour ce qui concerne le musicien classique, l'interprétation performative d'une représentation textuelle conduit et structure les attentes d'un public. Aussi bien l'interprète que le public peut ainsi faire confiance à un élément invariable par rapport à chaque performance, la partition, pour juger de la pertinence - mais pas toujours de l'efficacité - de l'exécution. Dans ce cas l'existence d'une mémoire délocalisée (ou désincarnée) fonctionne comme un lien. En ce qui concerne la performance improvisée, la mémoire intervient de façon différente. Il s'agit de la mémoire des performances précédentes (l'histoire performative d'un morceau) plutôt que de la mémoire délocalisée dans un texte. La dimension performative est ainsi connectée à la mémoire du corps. De plus, une telle mémoire est renégociée ex novo d'une performance à l'autre.

18. C. Cardew, "Towards an Ethics of Improvisation”, in Id., Treatise Handbook, Edition Pçeters, Londres 1971, p.47. Retourarticle18

19. Parfois cet abandon du corps se produit aussi dans le jazz. En effet lorsque le réalisateur Martin Ritt demande à Duke Ellington de composer la bande sonore du film Paris Blues, il lui laisse beaucoup de liberté mais en même temps Ritt insiste sur la nécessité d'avoir un son lisse et crémeux comme le son du blanc, Tommy Dorsey, et non pas growling et vocal comme la sonorité de la bande de Duke Ellington (au début du $\mathrm{XX}^{\mathrm{e}}$ siècle, le trombone était déjà un instrument sonore et " parlant ", un instrument qui servait aux clowns à produire des pets et des rots).

20. Harmonie, mélodie, rythme et timbre sont en fait des catégories et non pas des "paramètres", terme emprunté aux mathématiques et qui fait penser à des propriétés intrinsèques à la musique (mais qui révèle la prétention à en mesurer et contrôler tous les aspects). Il ne faut oublier qu'au Bas Moyen-Âge la musique était considérée comme l'une des arts du Quadrivium, avec l'arithmétique, la géométrie et l'astronomie.

21. Amiri Baraka (aka Le Roi Jones) dans "The Jazz avant-garde" (1961) in L. Jones, Black Music, Da Capo Press, New York, 1998, p.77 note que tous les joueurs de hanche sont fascinés par le son de la voix humaine parce que le jazz, à son avis, et la musique afro-américaine en général, est ancrée dans la voix et ne peut s'en éloigner trop.

22. Il faudrait d'ailleurs mettre en question l'idée vidéocentriste et " perfectionniste » que c'est la maximisation du principe de l'équilibre, de l'achèvement et de la complétude, l'unité organique de l'œuvre - dont la forme est la «meilleure» parce qu'elle n'a pas des parties superflues et des omissions significatives. Dans sa Théorie esthétique, Adorno parle de fermeture (Geschlossenheit), de connexion immanente (Immanenzzusammenhang) de cohérence (Stimmigkeit)de l'œuvre d'art (des tels critères conduiront Adorno à considérer le jazz comme responsable d'une Entkunstung, une désartification de la musique). Mais sommes-nous certains que le concept architectonique de forme soit utile pour rendre compte d'un processus improvisé (à savoir de l'événement qui en train de se dérouler)?

23. Dans les partitions de Cardew il y a beaucoup de contre-indications et d'écarts (saute ce passage, fais l'inverse de ce qui prescrit, ajoute quelque chose à ton gré) qui corrompent l'autorité incorporée dans la notation. 
24. Nous pensons aussi à Terry Riley dont la composition In $C$ propose un ensemble des petites cellules mélodiques qui s'étendent progressivement mais qui peuvent être jouées pour tout ensemble d'instruments et pour une durée non pré-établie ; ou bien à LaMonte Young qui à fondé le Theater of Eternal Music en 1964 pour expérimenter de la musique de très longue durée (il a aussi enregistré $15 \mathrm{~cd}$ où il syntonise le piano). Dans tous ces exemples la musique n'est pas écrite et n'est pas fermée (elle n'a pas un début et une fin).

25. J. Molino, "Il puro e l'impuro", in Enciclopedia della musica, vol.1, Einaudi, Torino, 2001, p. 1056-1057.

26. Arnold Schönberg, Traité d'harmonie (1978), Éd. Média Musique, 2008

27. Edgar Varèse, Ecrits, Paris, Christian Bourgois, 1983.

28. Au sujet de la phonosphére, Morton Feldman raconte d'avoir trouvé l'inspiration pour The King of Denmark pendant qu'à la plage il écoutait le bruit de fond d'enfants, de petites radios, de fragments de discours... Luc Ferrari, à son tour, compose un morceau de 21 minutes lié à l'enregistrement de sons produits dans une plage yougoslave dans les années 1970.

29. A. B. Spellman, Four jazz lives, University of Michigan Press, Ann Arbor, 2004.

30. Gertrude Stein, Picasso, (Paris, Floury, 1938), Christian Bourgois, Paris (1978), 2006.

31. Déjà Lester Young faisait glisser la note jusqu'au moment où le son devient le souffle qui est immédiatement au dessous du son (voir le début de I'm confessin' enregistré pour Verve en 1952 avec The Oscar Peterson Trio). Une volonté esthétique analogue guide Ornette Coleman vers le saxophone en plastique : «Because you could almost see the shape of the breath of a note. With the metal you can't, the breath just dissolves in the metal ", J. Litweiler, Ornette Coleman. A Harmolodic Life, Morrow, New York, 1992, p.46.

32. Tous les sons, d'ailleurs, enregistrent la structure interne de ce qui les produit. Remplissez d'eau un seau et il résonnera différemment

33. S. Hall, « What is this 'Black' in black popular culture ?, The black studies reader, Routledge , New York \& London, 2004, p.255-263

34. L'individualisation vaut aussi pour le tap propre au batteur, sa manière d'appuyer la baguette sur la cymbale. Nous pouvons distinguer les joueurs à partir de leur approche ou leur attaque du tempo : ceux qui jouent de façon anticipée par rapport à la mesure (on top of the pulse) comme Kenny Clarke, Roy Haynes, Billy Higgins ou Jimmy Cobb et ceux qui lay back derrière la mesure comme Philly Joe Jones, Art Blakey, Elvin Jones, Paul Motlan ou Tony Williams.

35. Roland Barthes, Le Grain de la Voix : Entretiens, 1962-1980, Éditions du Seuil, Paris, 1981.

36. Voyons par exemple le récit de Gunther Schuller sur Ornette Coleman qui en 1960 était allé chez Schuller pour prendre des « cours» de lecture et de notation musicale, Litweiler, op.cit., p. 94, : «I knew I had to think of some new, ingenious ways of breaking through this mental barrier that he had. It never quite happened. There was one incredible moment when I felt I was making good progress and he was beginning to understand - I felt there was a light going on in his brain». Cette fois Ornette commence à gémir, se lève en vitesse et cours vomir dans les toilettes pendant 10 minutes. Il revient en s'excusant, visiblement éprouvé, mais il ne se représentera plus aux cours. A Schuller d'en conclure : «I'm not a professional psychologist, but I think what happened was that he caught a glimpse of what I was talking about in terms of accurate reading and notation and it was so disturbing because it meant everything he had learned up to then was'wrong' ". La primitivisation du jazz et sa mise en discussion dans un cadre qui oppose civilisation et barbarie est le symptôme d'un discours « cultivé » et didactique qui considère le jazz comme une musique arriérée à « dépasser ». Le repositionnement discursif de la contribution afro-américaine arrive d'ailleurs par une autre stratégie, celle eurocentriste qui se déguise en syncrétisme. Lorsque André Hodeir fait référence à la «corruption féconde de l'art blanc» de la part du «folklore noir » non seulement il se rend à l'image primordialiste mais l'alimente de son côté en remarquant que c'est par le biais de la rencontre entre des éléments de la musique occidentale et le jazz que celui-ci nous devient familier et compréhensible, A. Hodeir, Les Mondes du Jazz, (1970), André 
Dimanche, Birdland, Marseille, 1993. De cette façon le jazz est réintégré dans le contexte de grandes conquêtes occidentales. Mais comme notent Carles et Comolli, le jazz n'a jamais été une musique œcuménique; au contraire il crée de la tension, conserve ses propres contradictions internes qui étaient à son origine : blesse, provoque scission et divisions et doit le faire pour garder sa vitalité.

37. F. Postif, Les grandes interviews de jazz hot, Editions de l'Instant, Paris, 1989.

38. Même un instrument « minimal» comme le triangle (le sifflet ou la sonnette) demande de l'investissement et de l'exercice pour que les gestes du joueur s'inscrivent dans un corps entraîné en tant que marqueurs musculaires que le joueur peut réactiver à son gré comme source de créativité sonore. Il ne faut pas croire non plus que l'apprentissage de la compétence nécessaire pour improviser ne soit que physique comme si aucun effort cognitif et discursif n'y était impliqué. Le petit appartement de l'arrangeur Gil Evans à l'angle entre la 55st et la 5th avenue à New York, par exemple, disposait d'un piano mural, un tourne-disque, beaucoup de disques et était bondé 24 heures sur 24 de musiciens de jazz qui écoutaient, faisait des comparaisons, transcrivaient des solos, discutaient, analysaient et évaluent ou se situer dans la communauté de musiciens jazz.

39. Cecil Taylor lui-même a toujours pratiqué une intense activité sportive (de la danse en particulier). Il commence souvent une performance en s'approchant lentement au piano avec des mouvements et des sons presque félins : une sorte de rituel préparatoire qui montre comment la performance improvisée est liée à un corps en mouvement.

40. A. B. Spellman, op.cit, p. 149.

41. Ibidem, p. 4.

42. Ibidem, p.36.

43. John Cage and Roger Reynolds: A Conversation, by Roger Reynolds and John Cage, Oxford University Press, 1979.

44. D. Fontaine, Sound, a film by Dick Fontaine, 1967.

45. Voici deux exemples: Imaginary Landscape $n 1$ (1939) fut composée pour tourne-disques à vitesse à variable (il n'existe donc que sous forme d'enregistrement). In Imaginary Landscape $n^{\circ} 4$ en revanche 12 radios et 24 exécuteurs croisent au hasard sons, bruit et parole sans l'intervention du compositeur. Cage utilise des radios, de l'électroménager, de l'eau, de la glace et de la vapeur. Dans la plupart de ces « compositions » l'on réalise le souhait de Cage de se passer même de l'idéateur.

46. Lorsque j'affirme que la tradition eurologique a "manqué le corps » je reprends librement le titre d'un chapitre de Le grain de la voix de Barthes. Je sais parfaitement que Cage est un auteur polyédrique et que le corps n'est certes pas absent de sa production (il suffit de voir les partitions pour piano associées à la danse de Merce Cunningham). Il ne serait pas correct d'affirmer que Cage composait en faisant abstraction de l'exécution et d'un exécuteur en chair et os dont la routine était même mise en question. Cage ne travaille pas sur un «musical incarné » comme ferait un musicien jazz, mais peut-être plutôt qu'affirmer qu'il a «manqué le corps ", il faudrait dire qu'il a tenté de le frapper, de le secouer, de le «vider» (même si ici nous allons vers l'idée épistémologiquement problématique d'une ouverture totale par rapport au sonore en tant que tel).

47. Il faut d'abord souligner qu'il n'y a pas une culture (ou une société) orale et une culture de l'écriture. Il existe en revanche un savoir socio-anthropologique qui, sur la base des ses pratiques d'écriture, définit « orale » une culture qui ne possède pas d'écriture alphabétique.

Deuxièmement, l'oralité est souvent pensée comme ce qui permet de mettre le jazz dans un lieu entièrement différent par rapport à la musique classique occidentale. Les choses toutefois ne sont pas si simples. Bien que parler d'une culture orale signifie désormais faire référence à quelque chose par sa négation (elle n'a pas d'écriture) «orale» ne signifie pas "activité qui n'utilise aucun médium » comme si l'usage à tout moyen écrit « portait atteinte » au musicien de 
jazz. Les disques (et le gramophone ont été par exemple fondamentaux). Charlie Parker ralentissait la vitesse du tourne-disque pour pouvoir syntoniser le sax ténor de Lester Young à son sax alto pour pouvoir transcrire les solos de Young. Les disques sont donc arrivés à représenter non pas des manuels mais au moins une sorte de littérature sonore de référence (qui valorise des aspects comme le timbre ou l'inflexion ou encore le sens du tempo - aspects qu'il est difficile de représenter sous forme écrite - les disques d'ailleurs exaltent indirectement le rôle du performer plus que le rôle du compositeur). De toute façon le jazz ne s'identifie pas à la seule oralité performative comme s'il ignorait l'écriture. Dans le chapitre 3 de Il corpo sonoro, Il Mulino, Bologna, 2007, je consacre mon intérêt à la modalité propre au jazz d'exercer le signifying sur ou à partir de morceaux notés en estompant la dichotomie entre orale et écrit. Il ne faut pas non plus lier écriture et capacité théorétique et analytique, comme si celle-ci n'était pas nécessaire pour l'acquisition orale des modèles sur lesquels l'improvisation se fonde (avec la circonstance aggravante de laisser entendre que les musiciens de jazz ne sont pas capables de saisir et encore moins de thématiser les aspects structurels de leur art/activité). La conviction que le jazz serait «égalitaire " parce qu'il est oral est aussi non fondée. Dans une pratique orale le prestige est associé à la réputation qui exerce un certain contrôle sur les novices, au moins comme modèle à émuler. Il y a donc des formes de stratification qui se prolongent dans la hiérarchie des instruments les plus prestigieux, l'ordre des solos et leur durée (live mais surtout en studio). In fine il ne faut pas oublier que dans la musique "classique " le chef d'orchestre, au cours des répétitions, donne ses directives a voce. De plus, la plupart de musiciens « classiques » ne passent pas leur temps à lire des partitions mais à écouter des exécutions. Il faut donc un nouveau cadre conceptuel qui soit capable d'éclaircir la manière dont un ensemble de ressources et de dispositifs - orales, écrits et électroniques - collaborent à la formation du musicien.

48. J. L. Nancy, A l'écoute, Galilée, Paris, 2002, p.7.

49. M. Heidegger, Wegmarken, Vittorio Klostermann Verlag, Frankfurt am Main, 1967

50. Id., «L'époque de l'image du monde ", in Id, Chemins qui ne mènent nulle part, Gallimard, Paris, 2006.

51. Olivia Mattis, "From Be-bop to Poo-wip : jazz influences in Varèse's Poème électronique”, in Edgar Varèse. Composer, sound sculptor, visionary, ed. F. Meyer \& H. Zimmerman, The Boydell Press, Woodbridge, Suffolk, p.309-317

\section{RÉSUMÉS}

En utilisant le concept de généalogie de Michel Foucault, cet article propose deux doubles thèses sur l'improvisation dans le jazz. La première est un diagnostic historique : une fois que la notion d'œuvre musicale écrite est devenue le paradigme esthétique pour évaluer la musique en général, la pratique orale de l'improvisation a été marginalisée. La seconde thèse est que la perception négative de l'improvisation est en relation avec les corps sonores qui la jouent. Tout langage implique un code phonétique : certains sons ne sont pas seulement admis mais aussi admirés, tandis que d'autres sont refusés et rejetés. La culture occidentale vidéocentriste et graphocentriste considère qu'un son ne peut être connu et compris que s'il est décomposé en segments visuels et/ ou écrits.

Ces thèses sont développées à travers l'analyse des différences ontologiques entre voir et écouter et des cas de John Cage et du jazz. Celui-ci en particulier montre que la relation fondamentale de l'improvisation avec la capacité d'exploiter la musique corporelle produite par la peau, la bouche, 
la langue, les lèvres, le torse, les bras, les mains des joueurs pour réaliser incessamment une communion entre musiciens et public

Relying on Michel Foucault's concept of genealogy, this paper offers two coupled thesis' concerning improvisation in jazz. The first is a historical diagnosis: once the notion of a musical «work» (written and through-composed) has become the aesthetic paradigm to evaluate music in general, the (oral) practice of improvisation has been marginalized. The second thesis is that improvisation has been perceived as threatening because of the way it is connected to the «sounding bodies» of those who enact it. Every language has an implicit phonetic code: some sounds are admitted (and admired), others are not. In a culture that emphasizes the visual and the written, a sound which does not fit in a code (say as part of the chromatic segmentation), hence a sound deprived of a semantic function, becomes threatening. This is shown by exploring the ontological differences between seeing and hearing, and by analyzing both the case of John Cage and of jazz. Especially jazz shows the extent to which improvisation is connected to the capacity to exploit the textural discrepancies between notes and sounds that go beyond specific musical notes (i.e., discrete and re-identifiable sounds, endowed with a name): it is the growl, the whine, the hard-hitting, edgy, raspy, coarse, harsh, guttural, hissing, screeching, broken, liquid, raucous - call it the grain of sound - that lends sound its affecting presence.

\section{INDEX}

Mots-clés : généalogie, improvisation musicale, jazz, oralité

\section{AUTEURS}

\section{DAVIDE SPARTI}

Davide Sparti , philosophe et sociologue italien, est professeur de sociologie de la communication et d'épistémologie des sciences sociales auprès de la faculté de Lettres de l'Université de Sienne. Il est auteur notamment de Identità e coscienza (2000), Wittgenstein Politico (2000), Epistemologia delle scienze sociali (2002), L'importanza di essere umani. Etica del riconoscimento (2003), Suoni inauditi. L'improvvisazione nel jazz e nella vita quotidiana (2005), Musica in nero (2007), Il corpo sonoro (2007).sparti@unisi.it 